\title{
"SELF-SCREENING" OF RHODOPSIN IN ROD OUTER SEGMENTS
}

\author{
Mathew Alpern, ${ }^{1, *}$ AnNe B. Fulton ${ }^{2}$ and Barbara N. Baker ${ }^{3}$ \\ 'Vision Research Laboratory, W. K. Kellogg Eye Center, University of Michigan, Ann Arbor, MI 48105, \\ U.S.A. ${ }^{2}$ Ophthalmology Department, Children's Hospital Medical Center, Boston, MA 02115, U.S.A. and \\ ${ }^{3}$ Institute of Molecular Biophysics, Florida State University, Tallahassee, FL 32306, U.S.A.
}

(Received 24 July 1986; in revised form 5 January 1987)

\begin{abstract}
Microspectrophotometry (MSP) shows rhodopsin highly concentrated (about $3.0 \mathrm{mmol} / \mathrm{l}$ ) in rod outer segments (ROS). Calculation of the in vivo absorption spectrum of human rhodopsin from such data reveals a striking failure to agree with the action spectrum of human rod vision. Agreement is good between the spectral distribution of absorption coefficients and the action spectrum, but the "concentration-broadening" (or "self-screening") introduced by the high end on absorbance at this concentration results in a misfit among the largest in the 93 years comparisons of this kind have been made! To deal with this anomaly, it has been suggested that "concentration-broadening" is inappropriate for rhodopsin in rod vision. This proposal was tested by comparing rod action spectra of 15-day-old and adult rats, since the lengths of ROS increase by a factor of about two in maturation. Three lines of evidence are inconsistent with it. Although the conundrum remains unexplained, it cannot be dismissed by supposing "self-screening" inappropriate for night vision.
\end{abstract}

Self-screening Rhodopsin Rod vision Electroretinogram Absorption spectrum

\section{INTRODUCTION}

The agreement between the absorption (or absorptance) spectrum of the human rod visual pigment (rhodopsin) and the action spectrum of human night vision became the fundamental datum in visual physiology upon its discovery by Koenig (1894). Though never perfect, this elegant convergence of results from molecular physical chemistry and experimental psychology compels the inference that the initial step linking the physical world to its visual perception by the human brain is photochemical.

Continued attempts to improve Koenig's approximate agreement followed as understanding deepened. Unfortunately, the agreement in the most recent comparison, estimating the rhodopsin absorption spectrum in situ from spectral analysis of human rod outer segments (ROS) with a microspectrophotometer (MSP) (Bowmaker and Dartnall, 1980), is almost as poor as that in the initial comparison.

\section{Theory}

To be seen, photons must be absorbed. If $I$ is the incident irradiance [photons of mono-

*To whom correspondence should be addressed. chromatic light (of wavenumber, or frequency, $m$ ) $\left.\mathrm{sec}^{-1} \mathrm{deg}^{-2}\right]$ and $I^{\prime}$ the irradiance absorbed, then

$$
\begin{aligned}
& I^{\prime}(m) / I^{\prime}\left(m_{0}\right) \\
= & I(m)\left[1-10^{-\alpha(m) c l}\right] / I\left(m_{0}\right)\left[1-10^{-\alpha\left(m_{0}\right) c l}\right]
\end{aligned}
$$

where $\alpha$ is the absorption coefficient of rhodopsin, $m_{0}$ is its wavenumber of peak absorption, $c$ is its concentration and $l$ the length of the light path. A plot of $I^{\prime}(m) I\left(m_{0}\right) / I^{\prime}\left(m_{0}\right) I(m)$ vs $m$ is an absorption spectrum. A plot of $I\left(m_{0}\right) / I(m)$ vs $m$ for some fixed effect is an action spectrum. Koenig implicitly assumed that at rod threshold, the amount of light absorbed is constant independent of wavelength. This assumption is now a corollary of a general principle of vertebrate photoreception: the principle of univariance (Naka and Rushton, 1966). It is evident from equation (1), assuming univariance, that if the wavenumber dependency of the effect is derived from absorption by rhodopsin alone, the two spectra will be the same.

The shape and the width of the absorption spectrum of a pigment varies with its concentration, as equation (1) makes clear. The higher the concentration, the broader the spectrum. The broadening of the absorption spectrum of a visual pigment with increase in its concen- 
tration is called "self-screening" (Brindley, 1960) or "concentration-broadening" (Bowmaker and Dartnall, 1980).

Bowmaker (1981) obtained a value of $0.018 \mu \mathrm{m}^{-1}$ for the specific absorbance [i.e. $\left.\alpha\left(m_{0}\right) c\right]$ of rhodopsin in a variety of species. MSP is most accurately employed with the measuring light passing transversely through the outer segment which is about $2 \mu \mathrm{m}$ in diameter. This dimension is sufficiently small that the higher order terms in the series expansion of the terms on the right hand side of equation (1) may safely be neglected. In that case, equation (1) reduces to

$$
I^{\prime}(m) I\left(m_{0}\right) / I^{\prime}\left(m_{0}\right) I(m)=\alpha(m) / \alpha\left(m_{0}\right)
$$

so the resulting data provide a measure of the spectral distribution of absorption coefficients. But in seeing, the light travels transversely down the rod which in a human ROS $26.5 \mu \mathrm{m}$ long leads to a full peak optical density [i.e. $\alpha\left(m_{0}\right) c l$ ] of 0.475 (or about $3.0 \mathrm{mmol}$ of rhodopsin/liter of rod volume). This is by no means a negligible density if the spectrum is computed by equation (1). Bowmaker and Dartnall (1980) found a poor fit of the action spectrum of human rod vision to the absorption spectrum computed from their MSP data by equation (1) (allowing for losses in the human lens) and "excellent agreement" with the absorption spectrum computed from the same data $\left(\lambda_{0}=497.6 \pm 3.3 \mathrm{~nm}\right)$ using equation (2).

To justify the latter comparison, they proposed that "... only the portion of the pigmentbearing rod membrane close to the inner segment is visually effective." This is consistent with the small directional sensitivity of rods compared with cones (Crawford, 1937; Stiles, 1939; Flamant and Stiles, 1948; Alpern et al., 1983) but it is inconsistent with ROS electrophysiology (Penn and Hagins, 1969; Baylor et al., 1979; Schnapf, 1983) as Bowmaker and Dartnall recognized. But there are other possibilities which do not share this inconsistency. Obviously, approximating the interaction of an array of rod outer segments with an incoming optical plane wave, neglecting the small diameter and high refractive index of the outer segments, and the presence of significant spaces between them, is a substantial simplification. Perhaps diffraction and scattering of light around the outer segments diminish their effective lengths as absorbers, so that the "selfscreening" effect is negligibly small. An alternative way of achieving the same result would be the loss of light by leakage out into the extracellular space in the course of its traverse down the ROS (Cornwall et al., 1984). A mechanism of this latter kind with oblique incidence of light on human cones is proposed, for example, as a component of the "self-screening" explanation of the Stiles-Crawford Color Effect (Walraven and Bouman, 1960; Enoch and Stiles, 1961).

The following experiments examine as a class theories which dismiss the relevance of "selfscreening" for rod vision. These experiments take advantage of the fact (cf. Fig. 1) that 15 days after birth, when a rat first opens his eyes, ROS are about half their adult length (Dowling and Sidman, 1962). If "self-screening" is not relevant for rod vision, the threshold action spectrum will be identical for infants and adults and equivalent to the spectral distribution of absorption coefficients of rat rhodopsin. "Selfscreening" predicts that the adult action spectrum will exhibit the characteristics of a spectrum with a peak density about $0.3 \log _{10}$ units larger than that of infants and will be substantially broader than the spectral distribution of absorption coefficients of rat rhodopsin.

\section{METHODS}

Albino (C-D strain, Charles River Breeding Labs) and pigmented Long-Evans rats-20 infants, age 15 days postnatal, and 23 adults, 2-6 months of age-were tested. They were maintained in a regimen of $10 \mathrm{hr}$ light, $14 \mathrm{hr}$ darkness. Animals, dark adpated overnight, were anesthetized with sodium pentobarbital $(5 \mathrm{mg} / 100 \mathrm{~g}$, i.p.). Pupils were dilated with atropine $1 \%$. The corneal and indifferent electrodes, mounted in a single speculum, were placed on the eye. 50 msec flashes with radiance controlled by neutral, and wavelength by interference, filters were exposed to the eye in the integrating sphere as previously described (Fulton and Graves, 1980). Electroretinogram (e.r.g.) voltages were amplified (direct coupled: $0-2 \mathrm{kHz}$ ), displayed on the face of a cathode ray oscilloscope and photographed. Stimulus/response curves were obtained for each animal for "monochromatic" lights in the spectral range $418-638 \mathrm{~nm}$ in approximately $20 \mathrm{~nm}$ steps. The spectral transmissivities of all filters were measured on a Cary 17 spectrophotometer; the radiances of unattenuated monochromatic lights were obtained with a calibrated silicon photosensitive diode (U.D.T. 500). Thresholds were expressed in terms of the number of pho- 

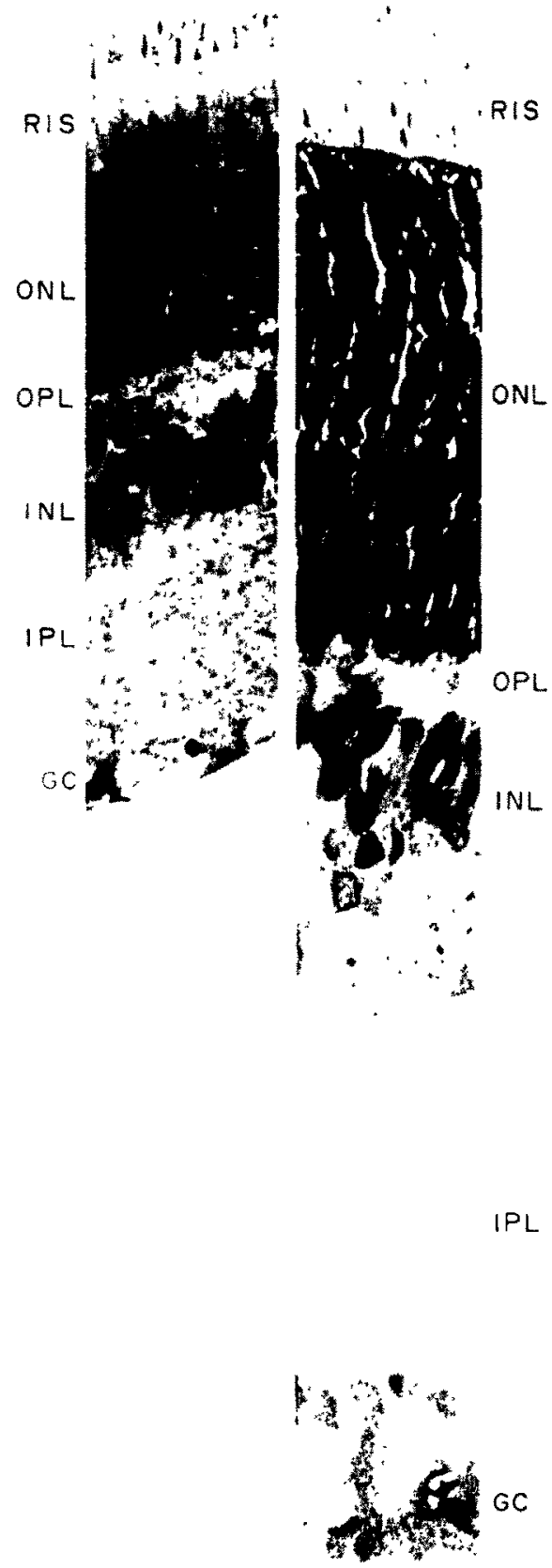

Fig. 1. Full thickness sections from equatorial regions of the retinas of 90 -day-old adult (left) and 15-day-old infant (right) rats as seen with a light microscope. The rod outer segments of adults are about twice the length found in infants. while the neural retina of the latter is $2.3 \times$ thicker than that of adults. RIS, rod inner segments; ONL, outer nuclear layer; $O P L$, outer plexiform layer; INL, inner nuclear layer; IPL, inner plexiform layer; GC, ganglion cell layer. The sections are $2 \mu \mathrm{m}$, stained with Toluidine blue; final magnification, $\times 700$. 
tons $(\lambda)$ incident (at the cornea) rod ${ }^{-1}$ assuming $15(10)^{6}$ rods/retina (Cone, 1963).

Similar action spectra were obtained from 10 adult pigmented Long-Evans rats in the dark following $5 \mathrm{~min}$ exposure to a ganzfeld radiance $6.3(10)^{16}$ equivalent photons $(503 \mathrm{~nm})$ $\mathrm{sec}^{-1} \mathrm{~cm}^{-2}$. Constraints on the radiance of the stimulus excluded the possibility of obtaining results at the spectral extremes during the earliest interval in the dark; the data under consideration were obtained between 40 and $60 \mathrm{~min}$ after the end of the bleach. The bleaching exposure reduced the concentration $c$ to $<0.05 \%$ of the dark concentration; during the recording interval from 75 to $50 \%$ of the rhodopsin remained bleached (Lewis, 1957; Dowling, 1963; Perlman, 1978).

For analysis, it is necessary to know the threshold number of photons incident on the rods (not on the cornea). To estimate lens losses, lenses were extracted from representative infant and adult eyes in warmed $\left(37^{\circ} \mathrm{C}\right)$ silicone oil to prevent cold cataract formation (in infant lenses). Lens and oil were placed in a single-well microscope slide which was then mounted on the microscope stage of the microspectrophotometer of Paul Brown in the Biological Laboratories of Harvard University. The spectral transmissivity of the lens plus oil was measured on this instrument (field stop set to a wide field), and the result was subtracted from that obtained from oil alone.

It was also necessary to correct for losses in the neural retina since (Fig. 1) in the equatorial region of the eye, the neural retina is more than twice as thick in infants as in adults (Braekevelt and Hollenberg, 1970). Sample pieces of retinas of each group were dissected from the equatorial region of the eye and gently shaken in physiological saline solution to remove ROS. The pieces of retinas in physiological saline were then bleached to remove any residual rhodopsin and a sample placed in a pool of fresh saline, receptor side up, on a flat microscope slide. Care was taken to select a region of each retinal specimen that appeared flat, free of blood vessels, and of uniform thickness. This slide was then mounted in the microscope stage of the same spectrophotometer and the specimen scanned from 700 to $310 \mathrm{~nm}$. The resulting scans were subtracted from the spectral scans of saline alone.

The results of these measurements of prereceptor absorbance at the action spectra wavenumbers were used to correct the thresholds at the cornea. They are plotted below in Fig. 2(A) for adult eyes and below in Fig. 2(B) for infant eyes. In each plot the diamonds connected by the dashed lines illustrate the absorbance of the neural retina while dots connected by the solid line show the total prereceptor absorbance (the absorbance of the lens is therefore the difference between the diamonds and dots). Three points about these data are noteworthy: (i) At $418 \mathrm{~nm}$ the mean density of adult lenses was $0.016 \log _{10}$ unit larger than that of infants; (ii) no obvious increase in absorbance of the neural retina was found in regions of the peak of rhodopsin, nor in regions of peaks of short-wavelength photoproducts; (iii) the mean density of the infant neural retina at $400 \mathrm{~nm}$ (not shown in Fig. 2(b)] $(0.37 \pm 0.0225)$ was found to be significantly higher than that mean density of adults $(0.281 \pm 0.0264, t=2.48,7$ d.f., $p<0.05)$.

To estimate the spectral distribution of rhodopsin absorption coefficients, ROS were isolated by sucrose flotation from both 16-week-old and 15-day-old albino rats. Retinas were removed in dim red light from darkadapated anesthetized animals. The retinas were gently homogenized in $40 \%$ sucrose and the homogenate overlaid with $0.067 \mathrm{M}$ phosphate buffer and spun at 12,000 r.p.m. for $20 \mathrm{~min}$ in an SS34 rotor of a Sorball RC-5 centrifuge. The material at the interface betwen the sucrose and the buffer was collected with a suction pipette, washed with buffer and centrifuged for $15 \mathrm{~min}$ at 12,000 r.p.m. The resultant pellet was resuspended in $35 \%$ sucrose by gentle homogenization and overlaid again with buffer and centrifuged as before. The material at the interface between the sucrose and buffer was collected with suction pipette, washed and centrifuged once more. The resultant pellet was then extracted with $15 \%$ Triton $\times 100$ in $0.067 \mathrm{M}$ phosphate buffer. Extraction continued for $3 \mathrm{~h}$ at room temperature with constant agitation. It has been previously determined that rat rhodopsin in $15 \%$ Triton X100 is thermally stable at room temperature (unpublished results). The absorption spectrum was measured in a Cary 14 spectrophotometer, first with ROS completely dark adapated and again after full bleaching in the presence of $0.1 \mathrm{~N} \mathrm{NH}_{2} \mathrm{OH}$. Three separate ROS preparations from three different retinas (one infant, two adult) yielded A400/A500 values of: $0.36,0.36$ and 0.33 . If, as these ratios suggest, the sample contained light and $\mathrm{NH}_{2} \mathrm{OH}$ insensitive products absorbing in the blue, they were present in both infant and adult ROS 


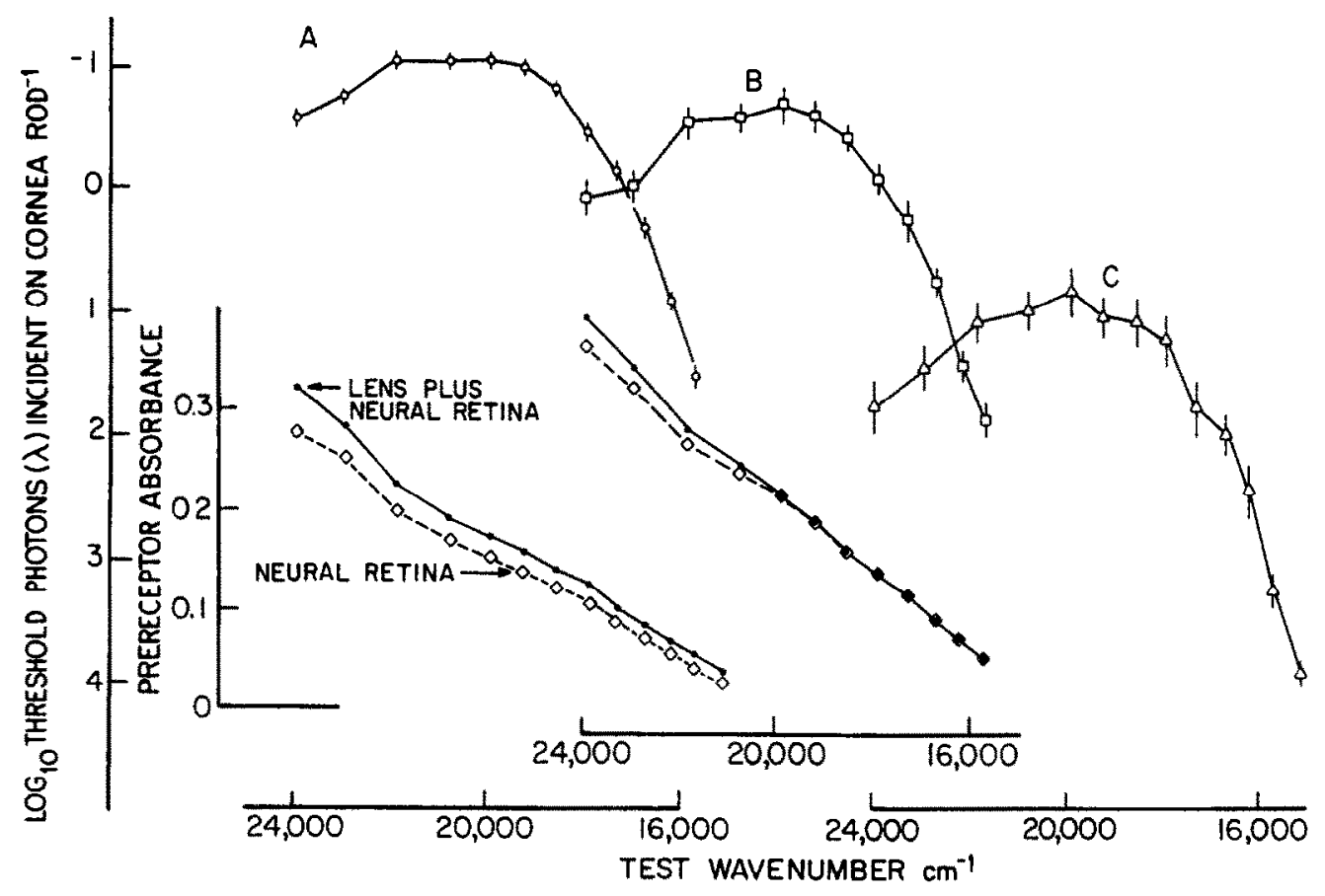

Fig. 2. Above mean ( \pm 1 SEM) log threshold [photons $(\lambda)$ incident at the cornea rod $^{-1}$ ] action spectra for $10 \mu \mathrm{v} b$-wave. (A) Circles are the average of dark-adapated thresholds for 23 adults. (B) Squares are thresholds of 20 dark-adapted infants. (C) Triangles are thresholds of 10 adults $40-60 \mathrm{~min}$ in the dark after bleaching $>95 \%$ of the rhodopsin in the retina. Note the shifting of the abscissa axis in the three spectra for clarity of the display. Below the prereceptor absorbances at the wavelengths for which the action spectra were tested. Diamonds connected by dashed lines are the absorbances of the neural retina; dots connected by a solid line are the total prereceptor absorbances (neglecting losses at the cornea and in the aqueous and vitreous humors). The absorbances in the lens are given by the differences between the dots connected by the solid line and diamonds connected with the dashed line.

preparations. Obviously difference spectra remain unaffected by such substances. The log of the absorbance difference spectrum is plotted as the ordinate as a function of wavenumber as filled circles at the bottom of Fig. 3 .

We tried to fit this difference spectrum with a modification of the Dartnall rhodopsin nomogram (Dartnall, 1953; Stiles and Wyszecki, 1974; Wyszecki and Stiles, 1982) with the minimization routine STEPIT (Chandler, 1965) following Pugh and Sigel (1978). The result of the application of this program (which shifts the template curve vertically and horizontally while simultaneously seeking the optimum density of rhodopsin at the wavenumber of maximum absorbance differences) is shown by the curve drawn with long dashes through the solid circles at the bottom of Fig. 3. Even not including the two highest frequencies $\left(m>22,857 \mathrm{~cm}^{-1}\right)$ which as part of a difference spectrum cannot fit an absorption spectrum template in any event because of short-wavelength absorbing photoproducts, the computer generated optimum fit of the Dartnall nomogram is only fair [root mean squares (r.m.s.) deviations of points from curve is 0.05021 ]. More important for the application of this template to the action spectra of this paper, the Dartnall nomogram has the wrong shape. Even at its best, it systematically overpredicts absorption for $m>m_{0}$ and systematically underpredicts absorption for $m<m_{0}$ when compared with the difference spectrum of albino rat rhodopsin. Consequently the wavenumber of peak absorbance of the computed curve is hypsochromically shifted compared with the peak of the best smooth curve fitted by eye to the same data set. This same procedure applied to the two other difference spectra of rat rhodopsin yielded the same results. For example, peak wavelengths $\left(\lambda_{0}=m_{0}^{-1}\right)$ of the curves fitted by computer to the three spectra were 493.2, 493.1 and $494.4 \mathrm{~nm}$; the r.m.s. deviations of the experimental points from these curves were $0.04995,0.05021$ and 0.03001 , respectively. The former values differ substantially from the peak of the absorption spectrum: 498.2 \pm $0.4 \mathrm{~nm}$ (Collins and Morton, 1950), $498 \pm 1 \mathrm{~nm}$ (Bridges, 1959), and of the difference spectrum, 


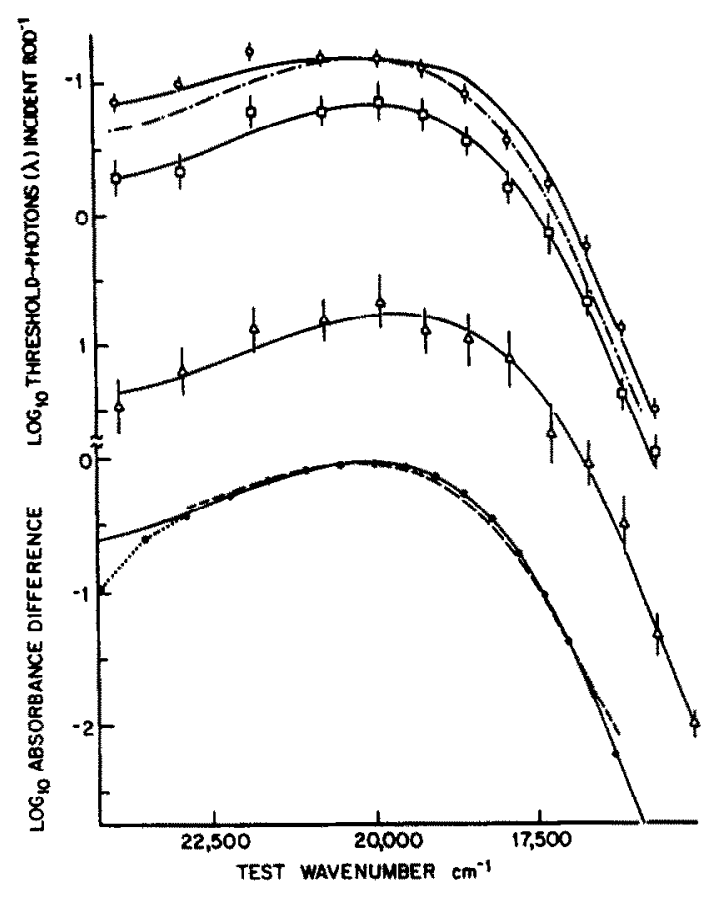

Fig. 3. Upper three sets of data are the mean ( \pm I SEM) thresholds photons $(\lambda)$ incident on the rods $\operatorname{rod}^{-1}$ replotted from the action spectra in Fig. 2 using the same symbols as in that figure (i.e. circles for dark-adapted adults, squares for dark-adapted infants, triangles, adults after bleaching) after correcting for losses in the lens and neural retina. The solid circles below are the absorbance, or optical density $[\alpha(m) c l]$ differences between fully dark-adapted and fully bleached rhodopsin. The plot of $\log$ absorbance difference has the same shape as the spectral distribution of absorption coefficients since change in concentration and path length merely displace the same curve vertically. The curve with the long dashes through this spectrum is the modified Dartnall nomogram (from frog rhodopsin) fitted by STEPIT to the data, ignoring the two points at the highest frequencies (connected by dotted lines) which cause the difference spectrum to deviate from the absorption spectrum because of short-wavelength absorbing photoproducts. The solid smooth curve is the STEPIT fit of the new template curve (derived as described in the text) to the same difference spectrum. It has the parameters itemized in parenthesis in Table 1. The dashed-dot curve is the solid curve (new template) fitted to the difference spectrum of rat rhodopsin shifted up to coincide with the peak of the dark-adapted adult threshold action spectrum.

$498.4 \pm 0.5 \mathrm{~nm}$ (Bridges, 1959) of rat rhodopsin, found in the literature. Evidently, the modified Dartnall nomogram, derived from frog rhodopsin, provides an unacceptable fit to spectra of rat rhodopsin.

The three differences spectra of rat rhodopsin were used to determine a new template. Each spectrum plotted as in Fig. 3 was fitted with a fifth-order polynomial for all $m \leqslant 22,857 \mathrm{~cm}$ for which the distortion by short wavelength ab- sorbing photoproducts can safely be neglected. The best fitting of these polynominals was then modified at the two spectral extremes to allow for extrapolation. At the short wave extreme ( $m=22,857 \mathrm{~cm}^{-1}$ ), the polynomial fit was equated with the absorption spectrum of the modified Dartnall nomogram and this curve replaced the polynomial for all larger wavenumbers. At the long wave extreme $\left(m \leqslant 16,327 \mathrm{~cm}^{-1}\right.$ ) the polynomial was replaced by a straight line (in this log linear plot) whose slope coincided with the slope at $16,327 \mathrm{~cm}^{-1}$ and whose ordinate value at that wavenumber was equal to that of the polynomial. It is not surprising that the resulting template [with a vertical scale factor, an horizontal scale factor and the density at the wavenumber at the peak, as curve-fitting parameters] yields a much closer description of the solid circles of Fig. 3, as the solid smooth curve (the result of this curvefitting procedure) drawn through them attests. The root mean square deviation of this fit is only 0.008 (neglecting, as before, the data at the two largest wavenumbers because of the distortion of the difference spectrum by short wavelength absorbing photoproducts). More important, the peak wavelength $\lambda_{0}=498.5 \mathrm{~nm}$ resulting from this fit is in excellent agreement with measurements of $\lambda_{0}$ of solutions of rat rhodopsin by Collins and Morton (1950) and by Bridges (1959).

\section{RESULTS}

The integrating sphere uniformly illuminates the entire retina. There is then no evidence for differences between the action spectra of pigmented and albino rats seen with smaller fields (Dodt, 1958; Dodt and Walther, 1958, 1959; Dodt and Echte, 1961). Hence, results from the two sets of animals have been pooled.

Mean threshold radiances $( \pm 1$ SEM) incident at the cornea, plotted as function of test wavenumber, are shown at the top of Fig. 2. Figure 2(A) shows the absolute thresholds of dark-adapted adults, Fig. 2(B) gives the threshold action spectrum for dark-adapted infants, while Fig. 2(C) shows the action spectrum of adults between 40 and $60 \mathrm{~min}$ in the dark after an exposure which bleached $>95 \%$ of the rhodopsin. Each individual action spectrum was corrected for losses in the lens and neural retina (i.e. all thresholds were converted to photons $(\lambda)$ incident on the rods rod ${ }^{-1}$ with the appropriate corrections for prereceptor absorbance shown 
Table 1. Parameters used in fitting a modified nomogram to rat action spectra for $b$-wave $(10 \mu v)$ threshold and to the rat rhodopsin difference spectrum

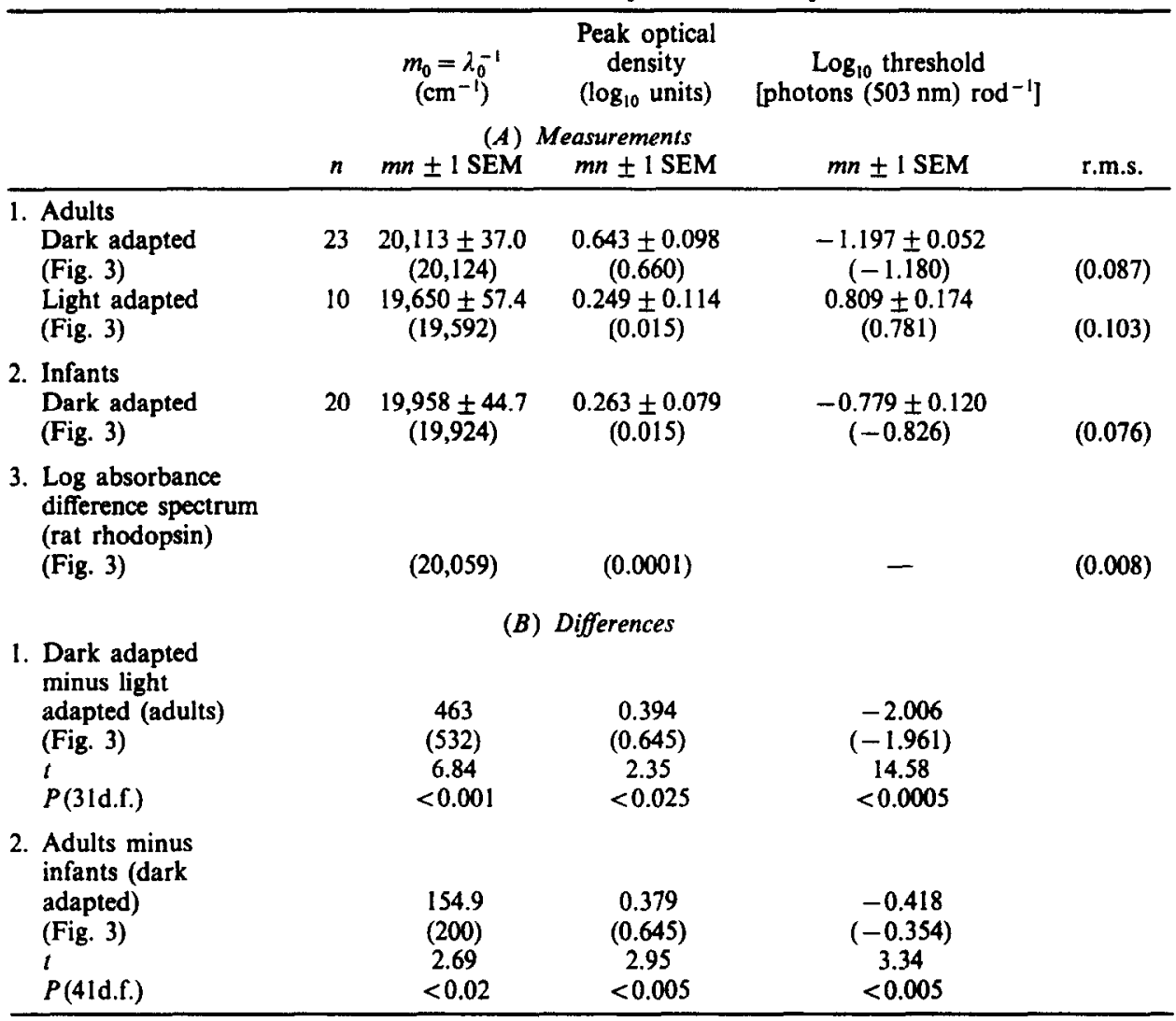

at the bottom of Figs $2(\mathrm{~A})$ and (B), neglecting losses at the cornea and in the aqueous and vitreous humors). The corrected three mean action spectra are plotted at the top of Fig. 3.

To obtain some estimates of the variability in parameters of these spectra, the new modified rhodopsin template developed as described in the "Methods" was substituted for the modified Dartnall nomogram in the STEPIT optimization routine as described by Pugh and Sigel (1978) to fit each individual corrected action spectrum (log threshold for $10 \mu \mathrm{v} b$-wave plotted as a function of test wavenumber) and to the mean action spectra illustrated in Fig. 3. There were three curve-fitting parameters available in optimizing these fits: (i) peak wavenumber $m_{0}$ (adjusting the horizontal position of the template curve); (ii) sensitivity at $503 \mathrm{~nm}$ (adjusting the vertical position of the template curve); (iii) the optical density at $m_{0}$ (adjusting the shape of the curve). In applying this routine, the peak density values available were constrained by the limits 1.32 and 0.015 [i.e. limits fixed by multiplying the largest adult ROS of Dowling and Sidman (1962) by three and the smallest infant
ROS they found by one third, assuming the specific absorbance $\left.0.018 \mu \mathrm{m}^{-1}\right]$. The average of the parameters from fitting individual curves are given first in Table $1(A)$ (mean \pm 1 SEM), while the parameters of the curves fitted to the average data in Fig. 3 are given beneath (in parentheses) along with the r.m.s. of the deviations of the average at each wavenumber, from the curve fitted through it. Statistical analysis of the differences in the means of the parameters of curves fitted to individual animals is summarized in Table $1(B)$.

Open circles in Fig. 3 show the mean corrected action spectrum of fully dark-adapted adults. At a test wavelength near the rhodopsin peak a $10 \mu \mathrm{v} b$-wave is achieved by a flash containing one photon $(503 \mathrm{~nm})$ incident upon every 15.7 rods. This is only slightly less sensitive than Cone (1963) found [one photon $(502 \mathrm{~nm})$ incident upon every 17.7 rods].

The squares in Fig. 3 display the mean action spectrum of 15-day-old rats. The threshold at peak sensitivity is considerably reduced; a $10 \mu \mathrm{v}$ $b$-wave is obtained with one photon ( $503 \mathrm{~nm}$ ) incident on every 6.0 infant rods. The insen- 
sitivity has several possible interpretations. The simplest is that the infant rod is less effective in catching photons because of the fewer rhodopsin molecules which it contains in comparison with the adult rod. If the difference is explained in this way alone, then ROS in the "average" adult animal are about 3.58 times longer than those of the "average" infant, assuming the average outer segment length found by Dowling and Sidman (1962) in 39-day-old animals and Bowmaker's estimate of specific absorbance. This is larger than the value (2.17 times) calculated from average infant ROS length (Dowling and Sidman, 1962). [The validity of this comparison rests on assumptions that: (i) the concentration of rhodopsin in the ROS remains unchanged in the two populations, (ii) there is not increase in length of ROS between 39 days and the 60 to 180-day age of our adult population, and (iii) there is no increase in the number of rat rods after 15 days. Evidence which can elucidate the validity of these assumptions is not extensive, but with regard to (iii), Denham (1967) found that in the developing rat retina, cell proliferation ceases after 14 days]. The agreement is closer with measurements of the ratio [2.9 (Dowling and Sidman, 1962) and 3.0 (Bonting et al., 1961)] of the amounts of extractable rhodopsin in the retinas of these two populations. On the whole, the agreement is reasonable.

Assuming univariance, the application of equation (1) with the relevant parameters predicts substatial differences in shapes between infant and adult action spectra. Although the mean results at 17,889 and $18,518 \mathrm{~cm}^{-1}$ fall substantially below and those at $21,834 \mathrm{~cm}^{-1}$ above the best fitting (i.e. solid smooth) curve fitted by STEPIT through the mean adult darkadapated spectra, a clear difference in the shapes of the expected kind between two spectra is qualitatively evident in Fig. 3 because these same anomalies (if somewhat smaller at 17,889 and $18,518 \mathrm{~cm}^{-1}$ ) are also apparent in the infant results. A more quantitatively satisfying verification of the effects of "self-screening" are found in the mean peak densities of the individual best fitting curves as shown in Table 1. The mean ( \pm 1 SEM) peak optical density of the curves fitted to the infant spectra was $0.263 \pm 0.079$. If every molecule of rhodopsin is equally effective and the specific absorbance reported by Bowmaker (1981) generalizes to rat, the peak optical density should be $0.200-0.232$ according to Dowling and Sidman's measure- ments of the average outer segment length in 15-day-old rods. Thus the agreement is good. On the other hand, the mean peak optical density of the curves fitted to the thresholds of dark-adapted adults $(0.643 \pm 0.098)$ is larger than comparable estimates from the average outer segment length of 39-day-old animals $(0.423)$. Given that this adult population is older than 39 days and the possibility that the outer segments of Dowling and Sidman's adult rods may not have reached their full length even at 39 days, the results are consistent with expectations of equation (1) applied to $b$-wave thresholds.

Changes in the peak optical density alter the adult action spectrum compared with the spectral distribution of absorption coefficients of rat rhodopsin as shown in Fig. 3. The smooth curve fitted to the log absorbance difference spectrum when shifted up to coincide with the peak of the action spectrum of dark-adapted adults is shown by the dashed-dot curve in this figure. This curve is much narrower than the solid curve fitted by computer to the open circles. With one reservation, differences between the two curves are characteristic of the predictions of "self-screening" if each rhodopsin molecule is equally effective and if the peak absorbance of adult rat ROS is about 0.64 .

The reservation in the preceding sentence is a very small (equivalent to about $1.3 \mathrm{~nm}$ wavelength difference) hypsochromic shift of $\bar{m}_{0}$ the average wavenumber of maximum sensitivity of the adult action spectrum compared with the absorbance difference spectrum of rat rhodopsin. The most plausible explanation is small measurement errors in the absorption spectra of the lens and neural retina used to correct the action spectra of adult animals or, what amounts to the same thing, minor differences between these spectra and their appropriate counterparts in the eyes of the animals studied at threshold. If that is the explanation, a similar discrepancy between the peak wavenumber of rat rhodopsin and that of the action spectrum of infant rats is not necessarily expected and indeed, the discrepancy in this case, though it is slightly larger (equivalent to a wavelength difference of about $2.5 \mathrm{~nm}$ ) is in the opposite direction to the shift in adults. According to this view, the $155 \mathrm{~cm}^{-1}$ difference in peak wavenumber of the action spectra of infant and adult animals, although significant statistically $(P<0.02)$ has a relatively trivial cause: inaccurate correction for losses in the ocular media. 
If "concentration-broadening" explains the broad action spectrum of adult rods, that spectrum can be expected to narrow after the rhodopsin has been bleached and its concentration in the outer segment is reduced. The triangles in Fig. 3 are adult thresholds obtained in the dark $40-60 \mathrm{~min}$ after $>95 \%$ of the rhodopsin had been bleached. They are qualitatively consistent with that expectation. On average, threshold after the bleach was elevated about $2.0 \log _{10}$ units above the dark-adapted threshold. Dowling (1960) found a $2.0 \log$ unit threshold elevation associated with a depletion of the retinal rhodopsin to $44 \%$ of its value in normal darkadapted rats. This is consistent with estimates of $25-50 \%$ regeneration at the moment in the dark these threshold measurements were made obtained from the time course of dark recovery of rhodopsin following bleaches of this magnitude in the normal rat retina (Lewis, 1957; Dowling, 1963; Perlman, 1978). The mean peak optical density obtained by curve fitting individual action spectra $(0.249 \pm 0.114)$ is slightly less than the value $(0.283)$ expected if the density obtained from averaging the peak absorbances of the curves fitted to the fully dark-adapted animals were reduced to $44 \%$ of the dark value by bleaching. But the agreement is reasonable.

"Self-screening" is not the only consideration in evaluating differences between dark thresholds measured before and after bleaching. The rat retina contains a small number of cones which cause a shift (to a smaller wavenumber) of $m_{0}$ of action spectra obtained in the dark after bleaching, when cone sensitivity has fully recovered while rod sensitivity remains depressed (Dodt and Echte, 1960; Green, 1971; Cicerone, 1976). Indeed, the shift in the peak of the adult action spectrum from $497.2 \pm 0.9 \mathrm{~nm}$ (dark adapted) to $508.9 \pm 1.5 \mathrm{~nm}$ (after bleaching) is almost certainly so explained. It is not possible to calculate an hypothetical action spectrum due to rods alone after this has been allowed for, because we know neither the relative numbers of three kinds of cones Cicerone (1976) found in this retina, nor how their sensitivities pool with rods after bleaching. So the matter remains open. In general, the results of these bleaching experiments are qualitatively consistent with "self-screening" applied to the proposition that every rhodopsin molecule in the rod is physiologically active and therefore with the differences in the action spectra found between dark-adapted adult and infant rats. A more quantitatively satisfying application of theory to thresholds after bleaching requires more quantitative information on the different species of rat cones, a better understanding of the pooling of rod and cone sensitivities in this animal as well as a more precise action spectrum after bleaching.

\section{DISCUSSION}

Three lines of evidence-(1) the absolute sensitivities of infant and adult rats, (2) the shapes of the action spectra at threshold in these two populations, and (3) the narrowing of this spectrum in adults after the concentration of rhodopsin has been reduced by bleaching-are all consistent with the theory of "self-screening" [equation (1)] applied to the excitation of rat rods. Although the quantitative agreements with published estimates of outer segment length and rhodopsin concentration are rarely perfect, these estimates are based upon relatively small samples, and the precision of quantitative generalizations from them, while unknown, is unlikely to be high. Measurement errors in those estimates and in the results of this paper may further reduce the accuracy of quantitative comparisons. But it seems quite impossible to deal even qualitatively with these three sets of results with no recourse to "selfscreening" theory.

The results of this paper show clearly enough that "self-screening" is relevant for rod vision of the rat at least. Might things be different for the night vision of people? Zwas and Alpern (1976), Baylor et al. (1984), and Nunn and Baylor $(1982,1983)$ offer evidence that it is not; but the contradiction introduced by Bowmaker and Dartnall's comparison is nonetheless real. The matter is analyzed further in the following paper (Alpern, 1987).

\section{SUMMARY}

1. The action spectra for absolute e.r.g. thresholds were compared in newborn and adult rats to examine the proposal that "selfscreening" by rhodopsin in the rods is not relevant for their night vision. Although the lengths of ROS double in maturation, this proposition predicts that the two spectra would be identical.

2. Three lines of evidence are inconsistent with this proposal. They support the alternative: that every molecule of rhodopsin is equally efficient in rod excitation. 
3. The Dartnall (1953) nomogram does not provide an adequate quantitative description of the difference spectrum of rat rhodopsin even in the part of the spectrum in which distortion from blue-absorbing photoproducts can safely be neglected. A new template has been derived.

Acknowledgements-Assisted by EY00197 to M.A. and EY05329 to A.B.F. We are especially grateful to Paul Brown for measuring the absorption spectra of the lenses and neural retinas. We also thank D. Barg, M. Cummings, R. Hansen, S. Harris, D. Kirk, A. Liepa, K. Manning and A. Osman for help in various ways.

\section{REFERENCES}

Alpern M. (1987) A note on the action spectrum of human rodvision. Vision Res. 27, 1471-1480.

Alpern M., Ching C. C. and Kitahara K. (1983) The directional sensitivity of retinal rods. J. Physiol., Lond. 343, 577-592.

Baylor D. A., Lamb T. D. and Yau K. W. (1979) The membrane current of single rod outer segments. J. Phys iol., Lond. 288, 589-611.

Baylor D. A., Nunn B. J. and Schnapf J. L. (1984) The photocurrent noise and spectral sensitivity of rods of the monkey Macaca fascicularis. J. Physiol., Lond. 357, 575-607.

Bonting S. L., Caravaggio L. L. and Gouras P. (1961) The rhodopsin cycle in the developing vertebrate retina. 1. Relation of rhodopsin content, electroretinogram and rod structure in the rat. Exp. Eye Res. 1, 14-24.

Bowmaker J. K. (1981) Visual pigments and colour vision in man and monkeys. J. R. Soc. Med. 74, 348-356.

Bowmaker J. K. and Dartnall H. J. A. (1980) Visual pigments of rods and cones in a human retina. J. Physiol., Lond. 298, 501-511.

Braekevelt C. R. and Hollenberg M. J. (1970) The development of the retinas of the albino rat. Am. J. Anat. 127, 281-301.

Bridges C. D. B. (1959) The visual pigments of some common laboratory mammals. Narure, Lond. 184, $1727-1728$.

Brindley G. S. (1960) The Physiology of the Retina and Visual Pathways, p. 217. Arnold, London.

Chandler J. P. (1965) STEPIT, Quantum chemistry exchange program. Indiana University, Bloomington, Ind.

Cicerone C. M. (1976) Cones survive rods in the lightdamaged eye of the albino rat. Science, N.Y. 194, 1183-1185.

Collins F. D. and Morton R. A. (1950) Studies on rhodopsin 1. Methods of extraction and the absorption spectrum. Biochem. J. 47, 3-10.

Cone R. A. (1963) Quantum relations of the rat electroretinogram. J. gen. Physiol. 228, 1267-1286.

Cornwall M. C., MacNichol E. F. Ir and Fein A. (1984) Absorptance and spectral sensitivity measurements of rod photoreceptors of the tiger salamander, Ambystome $i$ grinium. Vision Res. 24, 1651-1659.

Crawford B. H. (1937) The luminous efficiency of light entering the eye pupil at different points and its relation to brightness threshold measurements. Proc. $R$. Soc. 124B, 81-96.
Dartnall H. J. A. (1953) The interpretation of spectral sensitivity curves. Br. Med. Bull. 9, 24-30.

Denham S. (1967) A cell proliferation study of the neural retina in the two-day rat. J. Embryol. exp. Morphol. 18, 53-66.

Dodt E. (1958) Physical factors in the correlation of electroretinogram spectral sensitivity curves with visual pigments. Am. J. Ophthal. 46, 87-90.

Dodt E. and Echte K. (1961) Dark and light adaptation in pigmented and white rat as measured by electroretinogram threshold. J. Neurophysiol. 24, 427-445.

Dodt E. and Walther J. B. (1958) Spektrale Sensitivitaet und Blutreflexion. Pfluegers Arch. 266, 187-192.

Dodt E. and Walther J. B. (1959) Electroretinographische Messung der Spektralsensitivitaet von Albinoaugen bei direkter und diaskleraler Belichtung. Pfluegers Arch. 268, 435-443.

Dowling J. E. (1960) Night blindness, dark adapation and the electroretinogram. Am. J. Ophthal. 50, 875-887.

Dowling J. E. (1963) Neural and photochemical mechanisms of visual adaptation in the rat. J. gen. Physiol. 46, 1287-1301.

Dowling J. E. and Sidman R. L. (1962) Inherited retinal dystrophy in the rat. J. cell. Biol. 14, 73-110.

Enoch J. M. and Stiles W. S. (1961) The colour change of monochromatic light with retinal angle of incidence. Optica Acta 8, 329-358.

Flamant F. W. and Stiles W. S. (1948) The directional and spectral sensitivities of the retinal rods to adapting fields of different wavelengths. J. Physiol., Lond. 107, 187-202.

Fulton A. B. and Graves A. L. (1980) Background adaptation in developing rat retina: an electroretinographic study. Vision Res. 20, 819-826.

Green D. G. (1971) Light adaptation in the rat retina: evidence for two receptor mechanisms. Science, N.Y. 174, 598-600.

Koenig A. (1984) Ueber den menschlichen Sehpurpur und seine Bedeutung fuer das Sehen. S. B. Akad. Wiss. Berlin 1894, 577-598.

Lewis D. M. (1957) Regeneraton of rhodopsin in the albino rat. J. Physiol., Lond. 136, 624-631.

Naka K. I. and Rushton W. A. H. (1966) An attempt to analyse colour reception by electrophysiology. J. Physiol. $185,556-586$.

Nunn B. J. and Baylor D. A. (1982) Visual transduction in retinal rods of the monkey Macasa fascicularis. Nature, Lond. 229, 726-728.

Nunn B. J. and Baylor D. A. (1983) Visual transduction in single photoreceptors of the monkey Macaca fascicularis. In Colour Vision (Edited by Mollon J. D. and Sharpe L. T.), pp. 1-11. Academic Press, New York.

Penn R. D. and Hagins W. A. (1969) Signal transmission along retinal rods and the origin of the electroretinographic a-wave. Nature, Lond. 223, 201-205.

Perlman I. (1978) Kinetics of bleaching and regeneration of rhodopsin in abnormal (RCS) and normal albino rats in vivo. J. Physiol., Lond. 278, 141-159.

Pugh E. N. Jr and Sigel C. (1978) Evaluation of the candidacy of the $\Pi$-mechanisms of Stiles for colormatching fundamentals. Vision Res. 18, 317-330.

Schnapf J. L. (1983) Dependence of the single photon response on longitudinal position of absorption in toad rod outer segments. J. Physiol., Lond. 343, 147-159.

Stiles W. S. (1939) The directional sensitivity of the retina and the spectral sensitivities of the rods and cones. Proc. R. Soc. 127B, 64-105. 
Stiles W. S. and Wyszecki G. (1974) Colour-matching data and the spectral absorption curves of visual pigments. Vision Res. 14, 195-207.

Walraven P. L. and Bouman M. A. (1960) Relation between directional sensitivity and spectral response curves in human cone vision. J. opt. Soc. Am. 50, 780-784.
Wyszecki G. and Stiles W. S. (1982) Color Science: Concepts and Methods, Quantitative Data and Formulas, 2nd edn, pp. 591-604. Wiley, New York.

Zwas F. and Alpern M. (1976) The density of rhodopsin in the rods. Vision Res. 16, 121-127. 